\title{
Antimicrobial Susceptibility Pattern of Salmonella Isolates at Tertiary Care Hospital, Ahmedabad, India
}

\author{
Khyati Prajapati*, Mital Gamit and Mahendra Vegad \\ B.J. Mediacal College, Ahmedabad, Gujarat, India \\ *Corresponding author
}

\begin{tabular}{|c|}
\hline Keywords \\
\hline $\begin{array}{l}\text { Salmonella, } \\
\text { Antibiotics, } \\
\text { Susceptibility pattern, } \\
\text { Enteric fever }\end{array}$ \\
\hline Article Info \\
\hline $\begin{array}{l}\text { Accepted: } \\
\text { 02 May } 2018 \\
\text { Available Online: } \\
\text { 10 June } 2018\end{array}$ \\
\hline
\end{tabular}

A B S T R A C T

\begin{abstract}
Background and Objective: Enteric fever is a global health problem causing high morbidity and mortality, especially in endemic areas such as India. The problem is exacerbated as the causative agent, Salmonella enterica subspecies enterica serovar Typhi (S. typhi), rapidly develops resistance to drugs used in treatment. Factors responsible for emergence of new epidemic strains of Salmonella are not understood, but it is possible that antimicrobial play a role in emergence and persistence of epidemic MDR strains. The MDR $S$. typhi is on the rise. The object of this study was to determine the susceptibility pattern of various drugs used for treatment of enteric fever. Total of 1020 blood samples obtained from suspected enteric fever patients during June 2015 to July 2016. The sample was processed on Bact/ALERT 3D and isolates obtained from subculture were identified by morphological, biochemical and serological means. Antibiotic susceptibility testing was carried out using modified Kirby-Bauer disk diffusion method. Out of 1020, 26 Salmonella were isolated with isolation rate of $2.55 \% .21$ were Salmonella typhi and 5 were Salmonella paratyphi A. Susceptibility pattern of S. typhi isolates were $85.71 \%$ to ampicillin, $100 \%$ to chloramphenicol, $90.48 \%$ to co-trimoxazole and 3rd generation cephalosporins, $57.14 \%$ to fluoroquinolones. There was higher number of Salmonella enterica serotype typhi isolates resistant to fluoroquinolones (pefloxacin and ciprofloxacin). However chloramphenicol was sensitive to all isolates. This study suggests chloramphenicol as a drug of choice for enteric fever and further monitoring of efficacy of older and newer antibiotics are desirable.
\end{abstract}

\section{Introduction}

Enteric fever is a global public health problem and is endemic in many developing countries, including India (Mathu et al., 2011). Enteric fever is a systemic infection caused by the human adapted pathogens Salmonella enterica serotype Typhi (S. typhi) and S. enterica serotype Paratyphi (S. paratyphi) A, B and C (Crump and Mintz, 2010). Enteric fever remains one of the major public health issues globally, especially in Asia. According to recently revised global estimate, above 22 million cases of typhoid fever occur each year round the world while $90 \%$ of the sufferers are from the South East Asia (Rahman et al., 2011). It predominantly affects children and young adults (Wasfy et al., 2000) and if not treated appropriately has mortality rate of $30 \%$, whereas, with proper treatment the 
mortality reduces to as low as $0.5 \%$ (Stormaon et al., 1997).

Drug resistance is fast becoming a major problem in the management of this infection and the emergence of multi-drug resistance has great implications for the therapy, for example, patients infected with such strains are more ill at presentation, have a longer duration of illness and higher mortality. However, there are no pathognomic features to distinguish such infections from infections with fully sensitive $S$. typhi at presentation (Kumar and Gupta, 2007). Chloramphenicol resistance became established globally in the S. typhi population after 1972 on plasmids of incompatibility group Inc $\mathrm{H}$ and Multi drug resistance (defined as resistance to all the first line antibiotics used to treat typhoid fever, i.e. chloramphenicol, ampicillin and cotrimoxazole) has been endemic, particularly in Indian sub-continent and South East Asian countries since 1984 (Bhutta et al., 1991). Though initially, individual plasmids were known to code for multidrug resistance to each of these antibiotics, since 1988 a single plasmid was known to code for multidrug resistance. This plasmid belongs to incompatibility group $\mathrm{H} 11$ and is highly permissible. In addition to Multi Drug Resistance (MDR) S. typhi, now resistances to fluoroquinolones have emerged as the newer challenges to the treatment of typhoid fever (Shrikala et al., 1999). This study was undertaken to isolate and identify $S$. typhi and $S$. paratyphi and their antibiotic sensitivity pattern which are the major cause of enteric fever in the developing countries like India.

\section{Materials and Methods}

The study was carried out at B.J. Medical College, Civil Hospital, Ahmedabad during June 2015 to July 2016. Total of 1020 blood samples were obtained from patients suspected with enteric fever.

\section{Isolation of Salmonella}

The samples to be tested were inoculated in BacT/ALERT culture bottle which was inserted into the BacT/ALERT 3D for incubation at $37^{\circ} \mathrm{C}$ and periodic reading for 7 days. Each culture vial contains a colorimetric sensor which can detect $\mathrm{CO}_{2}$ produced by the growth of microorganism. The sensor was monitored by the instrument every 10 minutes for an increase in its reflected light, which was proportional to the amount of presence of growth. A positive reading indicated the presumptive presence of viable microorganism in the bottle. Once a positive culture bottle was detected, a Gram stain slide was prepared from the bottle and then a loopful of the positive blood culture bottle content was subcultured on blood agar, MacConkey agar, Chocolate agar and, incubated at $37^{\circ} \mathrm{C}$ for 18 24 hours.

\section{Identification and confirmation of Salmonella}

The colony growths were identified by colony characteristics and bio-chemical reactions like oxidase test, catalase test, triple sugar iron agar, IMViC (Indole, Methylered, Voges Proskauer and Citrate) test, amino acid decarboxylation test, sugar fermentation test etc. Final identification of isolates was confirmed serologically according to Kauffman-White classification using Salmonella (somatic and flagellar) antisera like Poly O, O-9, and H-d.

\section{Antibiotic sensitivity test}

The clinical isolates were subjected to antibiotic sensitivity test on Mueller-Hinton agar, using modified Kirby Bauer disc diffusion method as per Clinical Laboratory Standard Institute 2015 guidelines. The panel of antibiotics included were Ampicillin (10 $\mu \mathrm{g})$, Ciprofloxacin $(5 \mu \mathrm{g})$, Levofloxacin $(5 \mu \mathrm{g})$, 
Pefloxacin $(5 \mu \mathrm{g})$, Cefuroxime $(30 \mu \mathrm{g})$, Cefotaxime $(30 \mu \mathrm{g})$, Cefoperazone $(75 \mu \mathrm{g})$, Co-trimoxazole $(25 \mu \mathrm{g})$ and Chloramphenicol $(30 \mu \mathrm{g})$.

The negative samples were incubated for next seven days and then subcultured, before finally declaring them as culture negative.

\section{Results and Discussion}

Total of 26 Salmonella were isolated from 1020 blood samples of suspected enteric fever patients.16 isolates were from male patients and 10 isolates were from female patients.

Culture positive patients fell into age group of 0-45 yrs. 12 isolates were from children of age group 0 to 15 yrs. 14 isolates were from adults of age group 16 to 45 yrs. Out of 26 Salmonella isolates, 21 were Salmonella typhi and 5 were Salmonella paratyphi A as shown in Figure 1.

Antimicrobial susceptibility pattern of Salmonella typhi and Salmonella paratyphi during our study period has been described in Table 1 and 2 respectively.

In the last fifteen years, the emergence of resistance to the antibiotics has lead to large epidemics. Typhoid is now encountered mostly throughout the developing world. Management of this serious disease becomes complicated due to the resistance of antibiotics used for the treatment of enteric fever. It is becoming difficult to control the spread of multidrug resistant (MDR) Salmonella (Parry).

Out of 1020 blood samples, 26 Salmonella were isolated with isolation rate of $2.55 \%$ which is similar to study done by Nilesh Patel et al., (2015). In our study period, S. typhi outnumbered $S$. paratyphi A with almost 4 times higher rate of isolation in our region.

We have obtained blood samples from patients attending civil hospital, Ahmedabad. This hospital is referral medical hospital for the region and, many patients seek treatment here, after receiving some treatment at local level. This factor may interfere with organism isolation rate.

The highest incidence of typhoid occurs in 1645 years age group. Males are affected more frequently than females (Parry). In the present study, $14(53.85 \%)$ cases occurred between 16-45 years of age. $16(61.54 \%)$ isolates were from male patients and $10(38.46 \%)$ isolates were from female patients.

Table.1 Antibiotic susceptibility pattern of Salmonella typhi

\begin{tabular}{|c|c|c|}
\hline Antibiotic & Susceptible (\%) & Resistant (\%) \\
\hline Ampicillin (10ug) & $18(85.71 \%)$ & $3(14.29 \%)$ \\
\hline 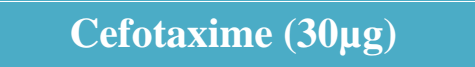 & $19(90.48 \%)$ & $2(9.52 \%)$ \\
\hline Cefoperazone $(\mathbf{7 5} \mu \mathrm{g})$ & $19(90.48 \%)$ & $2(9.52 \%)$ \\
\hline Ciprofloxacin $(5 \mu \mathrm{g})$ & $12(57.14 \%)$ & $9(42.86 \%)$ \\
\hline Pefloxacin $(5 \mu \mathrm{g})$ & $12(57.14 \%)$ & $9(42.86 \%)$ \\
\hline Co-trimoxazole (25 $\mu \mathrm{g})$ & $19(90.48 \%)$ & $1(4.26 \%)$ \\
\hline Chloramphenicol (30 $\mu \mathrm{gg})$ & $21(100 \%)$ & $0(0 \%)$ \\
\hline
\end{tabular}


Table.2 Antibiotic susceptibility pattern of Salmonella paratyphi A

\begin{tabular}{|c|c|c|}
\hline Antibiotic & Susceptible (\%) & Resistant (\%) \\
\hline Ampicillin (10ug) & $5(100 \%)$ & $0(0 \%)$ \\
\hline Cefotaxime (30 $\mu \mathrm{g})$ & $5(100 \%)$ & $0(0 \%)$ \\
\hline Cefoperazone $(75 \mu \mathrm{g})$ & $5(100 \%)$ & $0(0 \%)$ \\
\hline Ciprofloxacin (5 $\mu \mathrm{g})$ & $5(100 \%)$ & $0(0 \%)$ \\
\hline Pefloxacin (5 $\mu \mathrm{g})$ & $5(100 \%)$ & $0(0 \%)$ \\
\hline Co-trimoxazole (25 $\mu \mathrm{g})$ & $4(80 \%)$ & $1(20 \%)$ \\
\hline Chloramphenicol (30 $\mu \mathrm{g})$ & $5(100 \%)$ & $0(0 \%)$ \\
\hline
\end{tabular}

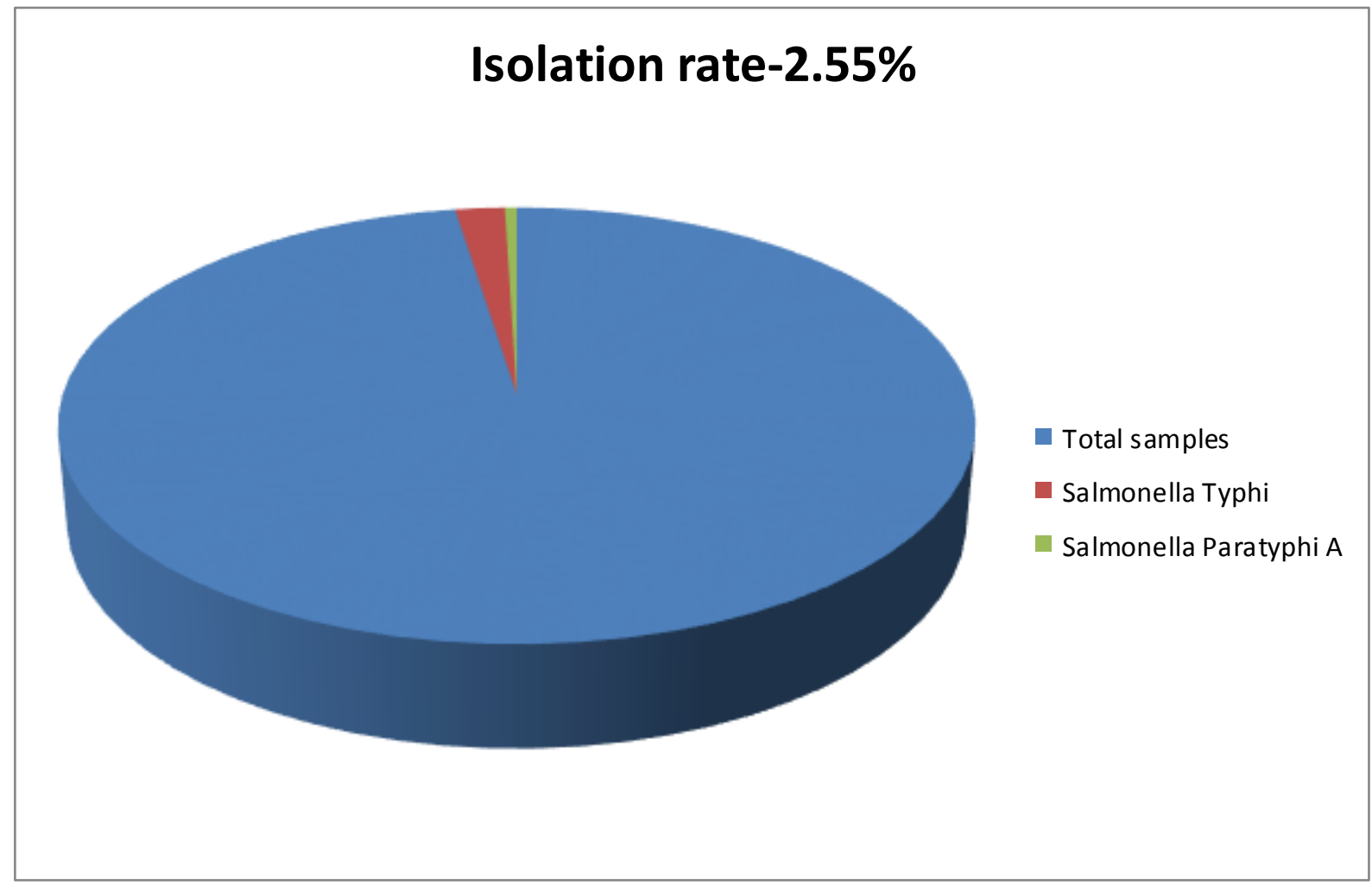

In our study, overall rate of resistance of $21 \mathrm{~S}$. typhi isolates was $14.29 \%$ to ampicillin, $9.52 \%$ to cephalosporins, $42.86 \%$ to fluoroquinolones and $4.76 \%$ to co-trimoxazole. Five isolates of $S$. paratyphi A shows high level of sensitivity to all antimicrobials.

S. paratyphi isolates were $100 \%$ sensitive to ampicillin, fluoroquinolones, co-trimoxazole chloramphenicol and $80 \%$ sensitive to $3^{\text {rd }}$ generation cephalosporins.

Susceptibility pattern of $S$. typhi isolates to ampicillin (85.71\%) and chloramphenicol $(100 \%)$ were similar to study conducted by Gordana Mijovic et al., (2012) whereas sensitivity to fluoroquinolones was $57.14 \%$ which was similar to study in Kuwait by Dimitrov et al., (2009). with sensitivity of $63.3 \%$.

Sensitivity of $S$. typhi isolates to co-trimoxazole and cephalosporins were $90.48 \%$ which was similar to study conducted by Gordana Mijovic et al., (2012). In conclusion, the findings of the 
present study indicated that the first line antibiotics may still have a role to play in the treatment of typhoid fever. This study suggests Chloramphenicol as a drug of choice for enteric fever and further monitoring of efficacy of older and newer antibiotics are desirable.

Sensitivity of Salmonella isolates to all tested antimicrobial agents except to fluoroquinolones was been good over testing period. Resistance rate to pefloxacin was higher and this fact deserves attention. The surveillance of antimicrobial resistance in Salmonella spp. is very important. Also, it is important to maintain Salmonella active surveillance of resistance on an international and intersectoral level.

\section{References}

Bhutta ZA, Naqvi SH, Razzaq RA, Farouqui BJ. Multidrug resistant in typhoid in children: Presentation and clinical features. Rev Infect Dis. 1991; 13: 83236.

Crump, J.A. and E.D. Mintz, 2010. Global trends in typhoid and paratyphoid fever. Clin. Infect. Dis., 50: 241-246. DOI: 10.1086/649541

Dimitrov T, Dashti AA, Albaksami O, Udo EE, Jadaon MM, Albert MJ: CiprofloxacinResistant Salmonella enterica Serovar Typhi from Kuwait with Novel Mutations in gyrA and parC Genes. Journal of Clinical Microbiology 2009; 47: 208211 19

Gordana Mijovic, Bogdanka Andric, Dragica Terzic, MilenaLopicic, Brankica Dupanovic, Montenegro antibiotic susceptibility of Salmonella spp.: a comparison of two surveys with a 5 years interval. JIMAB 2012, vol. 18, book 1

Kumar R, and Gupta NS. Multidrug-Resistant Typhoid Fever. Ind J Pediatr. 2007; 74(1): 39-42.

Mathu G, Suresh A, Sumathy G, Srivani R. (Studies on antimicrobial susceptibility pattern of Salmonella isolates from Chennai). India. Int. J. Pharma and Bio Sci, 2011; 2 (2): B-435-442.

Nilesh D. Patel, Rakesh M. Rajat, Rajesh S. Katara. In vitro antibiotic sensitivity pattern of Salmonella typhi. IAIM, 2015; 2(5): 1-4.

Parry CM. Epidemiological and clinical aspects of human typhoid fever. Salmonella Infections: Clinical, Immunological and molecular aspects. Ed. Pietro Mastroeni and Ducan Maskell. Cambridge University Press: pp. 1-15.

Rahman, A.K.M.M., M. Ahmad, R.S. Begum, M.Z. Hossain and S.A. Hoque et al., 2011. Prevalence of typhoid fever among the children in a semi urban area of Bangladesh. J. Dhaka Med. College, 20: 37-43. DOI: 10.3329/jdmc. v20i1.8570

Shrikala B, Shalini S, Vidyalakshmi K, Prathibha P. Ciprofloxacin resistance in Salmonella typhi. Natl Med J Ind. 1999; 12(3): 138 .

Stormaon MO, McIntyre PB, Morris J, Fasher B. Typhoid fever in children: diagnosis and therapeutic difficulties. Pediatr Infect Dis J. 1997; 16: 713-14.

Wasfy MO, Oyofo BA, David JC, Ismail TF, elGendy AM, Mohran ZS, et al., Isolation and antibiotic susceptibility of Salmonella, Shigella and Campylobacter from acute enteric infection in Egypt. J Health Popul. Nutr. 2000; 18: 33-38.

\section{How to cite this article:}

Khyati Prajapati, Mital Gamit and Mahendra Vegad. 2018. Antimicrobial Susceptibility Pattern of Salmonella Isolates at Tertiary Care Hospital, Ahmedabad. Int.J.Curr.Microbiol.App.Sci. 7(06): 156-160. doi: https://doi.org/10.20546/ijcmas.2018.706.018 